\title{
Quantization Dimension Estimate of Inhomogeneous Self-Similar Measures \\ by
}

\author{
Mrinal Kanti ROYCHOWDHURY
}

Presented by Stanistaw KWAPIEN

Summary. We consider an inhomogeneous measure $\mu$ with the inhomogeneous part a self-similar measure $\nu$, and show that for a given $r \in(0, \infty)$ the lower and the upper quantization dimensions of order $r$ of $\mu$ are bounded below by the quantization dimension $D_{r}(\nu)$ of $\nu$ and bounded above by a unique number $\kappa_{r} \in(0, \infty)$, related to the temperature function of the thermodynamic formalism that arises in the multifractal analysis of $\mu$.

1. Introduction. The term 'quantization' in this paper refers to the process of estimating a given probability by a discrete probability supported by a finite set. The quantization dimension $D_{r}$ of order $r$, where $r \in(0, \infty)$, of a probability measure is related to the asymptotic rate at which the expected distance (raised to the $r$ th power) to the support of the quantized version of the probability goes to zero as the support is allowed to go to infinity. This problem originated in information theory and some engineering technology such as image compression and signal processing (cf. [GN, Za]). Graf and Luschgy studied this problem systematically and gave a general mathematical treatment of it (cf. GL1]).

Given a Borel probability measure $\mu$ on $\mathbb{R}^{d}$, a number $r \in(0, \infty)$ and a natural number $n$, the $n$th quantization error of order $r$ of $\mu$ is defined by

$$
e_{n, r}:=e_{n, r}(\mu)=\inf \left\{\left(\int d(x, \alpha)^{r} d \mu(x)\right)^{1 / r}: \alpha \subset \mathbb{R}^{d}, \operatorname{card}(\alpha) \leq n\right\},
$$

where $d(x, \alpha)$ denotes the distance from the point $x$ to the set $\alpha$ with respect to a given norm $\|\cdot\|$ on $\mathbb{R}^{d}$. We note that if $\int\|x\|^{r} d \mu(x)<\infty$ then there is

2010 Mathematics Subject Classification: Primary 28A80; Secondary 60D05, 94A15.

Key words and phrases: quantization dimension, inhomogeneous self-similar measure, temperature function. 
some set $\alpha$ for which the infimum is achieved (cf. [GL1]). The upper and the lower quantization dimensions of order $r$ of $\mu$ are respectively defined by

$$
\bar{D}_{r}(\mu):=\limsup _{n \rightarrow \infty} \frac{\log n}{-\log e_{n, r}} \quad \underline{D}_{r}(\mu):=\liminf _{n \rightarrow \infty} \frac{\log n}{-\log e_{n, r}} .
$$

If $\bar{D}_{r}(\mu)$ and $\underline{D}_{r}(\mu)$ coincide, we call the common value the quantization dimension of $\mu$ of order $r$ and denote it by $D_{r}(\mu)$.

Let $S_{1}, \ldots, S_{N}$ be a set of contractive similarity mappings on $\mathbb{R}^{d}$ with similarity ratios $s_{1}, \ldots, s_{N}$ for $N \geq 2$. Then by $[\mathrm{H}]$, for a given probability vector $\left(p_{1}, \ldots, p_{N}\right)$ there exist a unique probability measure $\nu$, known as the self-similar measure, and a unique nonempty compact subset $E$ of $\mathbb{R}^{d}$, known as the self-similar set, satisfying the conditions

$$
\nu=\sum_{j=1}^{N} p_{j} \nu \circ S_{j}^{-1} \text { and } E=\bigcup_{j=1}^{N} S_{j}(E) .
$$

The iterated function system $\left\{S_{1}, \ldots, S_{N}\right\}$ satisfies the open set condition (OSC) if there exists a bounded nonempty open set $U \subset \mathbb{R}^{d}$ such that $\bigcup_{j=1}^{N} S_{j}(U) \subset U$ and $S_{i}(U) \cap S_{j}(U)=\emptyset$ for $1 \leq i \neq j \leq N$. Furthermore, the system satisfies the strong open set condition (SOSC) if $U$ can be chosen such that $U \cap E \neq \emptyset$, and the strong separation condition (SSC) if $S_{j}(E)$ are pairwise disjoint for $j=1, \ldots, N$. Note that in the case of similarity mappings with a finite number of mappings, the open set condition implies the strong open set condition (cf. [S] ). If $\left\{S_{1}, \ldots, S_{N}\right\}$ satisfies the strong separation condition then - as is easily seen-it also satisfies the open set condition, and hence the strong open set condition.

Under the open set condition, Graf and Luschgy showed that the quantization dimension function $D_{r}:=D_{r}(\nu)$ of the probability measure $\nu$ exists, and satisfies the following relation (cf. [GL1, GL2]):

$$
\sum_{j=1}^{N}\left(p_{j} s_{j}^{r}\right)^{D_{r} /\left(r+D_{r}\right)}=1 .
$$

In addition, they proved a stronger result:

$$
0<\liminf n e_{n, r}^{D_{r}}(\nu) \leq \lim \sup n e_{n, r}^{D_{r}}(\nu)<\infty .
$$

From (1) it is clear that the quantization dimension function of a self-similar probability measure is related to the temperature function of the thermodynamic formalism.

Let $S_{j}: \mathbb{R}^{d} \rightarrow \mathbb{R}^{d}$ be contractive similarities with similarity ratios $s_{j}$ for $j=1, \ldots, N, N \geq 2$. Also, let $\left\langle p_{j}\right\rangle:=\left(p_{0}, p_{1}, \ldots, p_{N}\right)$ be a probability vector and $\nu$ be a probability measure on $\mathbb{R}^{d}$ with compact support $E$. Then there exists a unique probability measure $\mu$ with compact support $K_{E}$ such 
that

$$
\mu=p_{0} \nu+\sum_{j=1}^{N} p_{j} \mu \circ S_{j}^{-1} \text { and } K_{E}=\bigcup_{j=1}^{N} S_{j}\left(K_{E}\right) \cup E .
$$

Here $\mu$ is called the inhomogeneous self-similar measure, and $K_{E}$ is called the inhomogeneous self-similar set associated with the list $\left(S_{1}, \ldots, S_{N}, p_{0}, p_{1}\right.$, $\left.\ldots, p_{N}, \nu\right)$. For details about inhomogeneous self-similar sets and measures one could see OS1, OS2, OS3]. Following [B, L], we also call $\left(\left\{S_{j}\right\},\left\langle p_{j}\right\rangle, \nu\right)$ a condensation system, $\mu$ the attracting measure and the set $K_{E}$ the attractor of the condensation system $\left(\left\{S_{j}\right\},\left\langle p_{j}\right\rangle, \nu\right)$.

In this paper, we will focus on condensation systems for which $\nu$ is a self-similar measure associated with the system of self-similar mappings $\left\{S_{1}, \ldots, S_{N}\right\}$ and a probability vector $\left(t_{1}, \ldots, t_{N}\right)$, i.e., $\nu$ satisfies $\nu=$ $\sum_{j=1}^{N} t_{j} \nu \circ S_{j}^{-1}$, where $t_{j}>0$ for $1 \leq j \leq N$. Let $E$ be the support of $\nu$. Then for the support $K_{E}$ of the attracting measure $\mu$, by the uniqueness of the compact set $K_{E}$, we must have $K_{E}=E$. Under the strong separation condition, Zhu [Zh] determined the lower and the upper quantization dimensions of the attracting measure $\mu$.

In this paper, under the strong separation condition we prove that for a given $r \in(0, \infty)$ there exists a unique $\kappa_{r} \in(0, \infty)$ such that

$$
D_{r}(\nu) \leq \underline{D}_{r}(\mu) \leq \bar{D}_{r}(\mu) \leq \kappa_{r},
$$

where $\underline{D}_{r}(\mu)$ and $\bar{D}_{r}(\mu)$ are respectively the lower and the upper quantization dimensions of order $r$ of $\mu$, and $D_{r}(\nu)$ is the quantization dimension of the associated self-similar measure $\nu$. Moreover, we show that $\kappa_{r}$ is related to the temperature function $\beta(q)$ of the thermodynamic formalism that arises in the multifractal analysis of $\mu$, namely $\kappa_{r}=\beta\left(q_{r}\right) /\left(1-q_{r}\right)$ where $\beta\left(q_{r}\right)=r q_{r}$.

2. Basic definitions, lemmas and propositions. In this paper, $\mathbb{R}^{d}$ denotes the $d$-dimensional Euclidean space equipped with a metric $d$ compatible with the Euclidean topology. Let us write

$$
\begin{aligned}
& V_{n, r}(\mu)=\inf \left\{\int d(x, \alpha)^{r} d \mu(x): \alpha \subset \mathbb{R}^{d}, \operatorname{card}(\alpha) \leq n\right\}, \\
& u_{n, r}(\mu)=\inf \left\{\int d\left(x, \alpha \cup U^{c}\right)^{r} d \mu(x): \alpha \subset \mathbb{R}^{d}, \operatorname{card}(\alpha) \leq n\right\},
\end{aligned}
$$

where $U$ is a set which comes from the open set condition and $U^{c}$ denotes its complement. We see that

$$
u_{n, r}^{1 / r} \leq V_{n, r}^{1 / r}=e_{n, r} .
$$

We call sets $\alpha_{n} \subset \mathbb{R}^{d}$ for which the above infimums are achieved $n$-optimal sets for $e_{n, r}, V_{n, r}$ or $u_{n, r}$ respectively. If $n=1$, we simply write $e_{r}, V_{r}$ or $u_{r}$. 
As stated above, Graf and Luschgy have shown that $n$-optimal sets exist when $\int\|x\|^{r} d \mu(x)<\infty$.

Let $\Omega=\{1, \ldots, N\}$. Let $\Xi_{0}$ denote the set consisting of only the empty word $\emptyset$. We define

$$
\Xi_{n}:=\prod_{k=1}^{n} \Omega, \quad \Xi_{*}:=\bigcup_{k=0}^{\infty} \Xi_{k}, \quad \Xi:=\prod_{k=1}^{\infty} \Omega .
$$

For any $\sigma \in \Xi_{*}$, if $\sigma=\sigma_{1} \cdots \sigma_{k} \in \Xi_{k}$ we call $k$ the length of $\sigma$, denoted by $|\sigma|$; the length of the empty word is zero. If $\sigma \in \Xi$ then $|\sigma|=\infty$. For any $\sigma \in \Xi_{*} \cup \Xi$ with $|\sigma| \geq n \geq 1$, we write $\left.\sigma\right|_{n}$ for the initial segment of $\sigma$ of length $n$, i.e., $\left.\sigma\right|_{n}:=\sigma_{1} \cdots \sigma_{n}$, and $\left.\sigma\right|_{0}=\emptyset$. If $\sigma, \tau \in \Xi_{*}$ and $|\sigma| \leq|\tau|$, $\sigma=\left.\tau\right|_{|\sigma|}$, we call $\sigma$ a predecessor of $\tau$ and write $\sigma \prec \tau$; if $\sigma \nprec \tau$ and $\tau \nprec \sigma$, we say $\sigma$ and $\tau$ are incomparable. For any two words $\sigma=\sigma_{1} \cdots \sigma_{k}$ and $\tau=\tau_{1} \cdots \tau_{p}$ in $\Xi_{*}$, by $\sigma * \tau:=\sigma \tau$ we mean the concatenation of $\sigma$ and $\tau$, i.e., $\sigma \tau=\sigma_{1} \cdots \sigma_{k} \tau_{1} \cdots \tau_{p}$. For $n \geq 2$ and $\sigma=\sigma_{1} \cdots \sigma_{n} \in \Xi_{n}$ we define $\sigma^{-}:=\sigma_{1} \sigma_{2} \cdots \sigma_{n-1}$.

Throughout the paper, $S_{1}, \ldots, S_{N}$ represent self-similar mappings with similarity ratios $s_{1}, \ldots, s_{N}$ respectively. Let $\nu$ be the self-similar measure associated with the probability vector $\left(t_{1}, \ldots, t_{N}\right)$, i.e., $\nu$ satisfies $\nu=\sum_{j=1}^{N} t_{j} \nu$ $\circ S_{j}^{-1}$, where $t_{j}>0$ for $1 \leq j \leq N$; let $\mu$ be the attracting measure of the condensation system $\left(\left\{S_{j}\right\},\left\langle p_{j}\right\rangle, \nu\right)$ as defined in the previous section, and $E$ be its attractor. Write

$$
s_{\max }=\max \left\{s_{1}, \ldots, s_{N}\right\} .
$$

If $\sigma$ is the empty word we write $E_{\sigma}=E$, and if $\sigma=\sigma_{1} \cdots \sigma_{n} \in \Xi_{n}, n \geq 1$, then

$$
S_{\sigma}:=S_{\sigma_{1}} \circ \cdots \circ S_{\sigma_{n}}, \quad E_{\sigma}:=S_{\sigma}(E), \quad s_{\sigma}:=s_{\sigma_{1}} \cdots s_{\sigma_{n}} .
$$

Each set $E_{\sigma}$ for $\sigma \in \Xi_{*}$ is called a cylinder set. If $S_{1}, \ldots, S_{N}$ satisfy the strong separation condition, then it is easy to see that $\mu\left(E_{\sigma} \cap E_{\tau}\right)=0$ for any two incomparable words $\sigma$ and $\tau$ in $\Xi_{*}$.

Inductively, let us define functions $g^{(1)}, g^{(2)}: \Xi_{*} \rightarrow \mathbb{R}$ as follows: If $\sigma=i$, where $1 \leq i \leq N$, set

$$
g^{(1)}(i):=p_{0} t_{i}, \quad g^{(2)}(i):=p_{i} .
$$

Now suppose $g^{(1)}(\sigma)$ and $g^{(2)}(\sigma)$ are defined for all words $\sigma \in \Xi_{k}$ for some $k \geq 1$. For $\tau \in \Xi_{k+1}$, we have $\tau^{-} \in \Xi_{k}$ and $\tau=\tau^{-} * i$ for some $i=1, \ldots, N$. We define

$$
g^{(1)}(\tau)=g^{(1)}\left(\tau^{-}\right) t_{i}+g^{(2)}\left(\tau^{-}\right) p_{0} t_{i} \quad \text { and } \quad g^{(2)}(\tau)=g^{(2)}\left(\tau^{-}\right) p_{i} .
$$

Thus $g^{(1)}(\tau)$ and $g^{(2)}(\tau)$ are well defined for $\tau \in \Xi_{k+1}$. Hence $g^{(1)}(\tau)$ and $g^{(2)}(\tau)$ are well defined for all words $\tau \in \Xi_{*}$. For the attracting measure $\mu$, 
by (3) we have

$$
\mu=p_{0} \nu+\sum_{j=1}^{N} p_{j} \mu \circ S_{j}^{-1}=\sum_{j=1}^{N}\left(p_{0} t_{j} \nu \circ S_{j}^{-1}+p_{j} \mu \circ S_{j}^{-1}\right) .
$$

Then using (4), by induction, for any $n \geq 1$ we can prove

$$
\mu=\sum_{|\tau|=n}\left(g^{(1)}(\tau) \nu \circ S_{\tau}^{-1}+g^{(2)}(\tau) \mu \circ S_{\tau}^{-1}\right) .
$$

Hence for $\sigma \in \Xi_{*}$, we have $\mu\left(E_{\sigma}\right)=g^{(1)}(\sigma)+g^{(2)}(\sigma)$, and for $\sigma \in \Xi_{n}, \tau \in \Xi_{p}$ with $n, p \geq 1$, we have

$$
\mu\left(E_{\sigma \tau}\right)=g^{(1)}(\sigma) \nu\left(E_{\tau}\right)+g^{(2)}(\sigma) \mu\left(E_{\tau}\right) .
$$

Let us now prove the following lemma.

Lemma 2.1. For any $\sigma, \tau \in \Xi_{*}$, we have

$$
\mu\left(E_{\sigma \tau}\right) \leq p_{0}^{-1} \mu\left(E_{\sigma}\right) \mu\left(E_{\tau}\right) .
$$

Proof. For $\sigma=j$ where $1 \leq j \leq N$, since $\mu\left(E_{\sigma}\right)=p_{0} t_{j}+p_{j} \geq p_{0} \nu\left(E_{\sigma}\right)$, we have

$$
\nu\left(E_{\sigma}\right) \leq p_{0}^{-1} \mu\left(E_{\sigma}\right) .
$$

For $\sigma=\sigma_{1} \cdots \sigma_{k} \in \Xi_{*}$ where $k>1$, set $\sigma^{b}=\sigma_{2} \cdots \sigma_{k}$. Then

$$
\mu\left(E_{\sigma}\right)=g^{(1)}\left(\sigma_{1}\right) \nu\left(E_{\sigma^{b}}\right)+g^{(2)}\left(\sigma_{1}\right) \mu\left(E_{\sigma^{b}}\right)=p_{0} t_{\sigma_{1}} \nu\left(E_{\sigma^{b}}\right)+p_{\sigma_{1}} \mu\left(E_{\sigma^{b}}\right),
$$

which implies $\mu\left(E_{\sigma}\right) \geq p_{0} \nu\left(E_{\sigma}\right)$, i.e., $\nu\left(E_{\sigma}\right) \leq p_{0}^{-1} \mu\left(E_{\sigma}\right)$. Thus for any two words $\sigma, \tau \in \Xi_{*}$, we have

$$
\begin{aligned}
\mu\left(E_{\sigma \tau}\right) & \leq g^{(1)}(\sigma) p_{0}^{-1} \mu\left(E_{\tau}\right)+g^{(2)}(\sigma) \mu\left(E_{\tau}\right) \\
& \leq p_{0}^{-1}\left(g^{(1)}(\sigma)+g^{(2)}(\sigma)\right) \mu\left(E_{\tau}\right) \leq p_{0}^{-1} \mu\left(E_{\sigma}\right) \mu\left(E_{\tau}\right)
\end{aligned}
$$

For $q, t \in \mathbb{R}$, let us define the auxiliary function

$$
Z_{k}(q, t)=\sum_{|\sigma|=k} \mu\left(E_{\sigma}\right)^{q} s_{\sigma}^{t}, \quad k \geq 1 .
$$

Then for the condensation system $\left(\left\{S_{j}\right\},\left\langle p_{j}\right\rangle, \nu\right)$ with attracting measure $\mu$, the topological pressure $P(q, t)$ is defined as follows:

$$
P(q, t)=\lim _{k \rightarrow \infty} \frac{1}{k} \log Z_{k}(q, t)=\lim _{k \rightarrow \infty} \frac{1}{k} \log \sum_{|\sigma|=k} \mu\left(E_{\sigma}\right)^{q} s_{\sigma}^{t},
$$

where $q, t \in \mathbb{R}$. The limit above exists by the standard theory of subadditive sequences (cf. [F1]), since for $n, p \geq 1$ and $q, t \in \mathbb{R}$, by Lemma 2.1, we have

$$
Z_{n+p}(q, t) \leq p_{0}^{-|t|} Z_{n}(q, t) Z_{p}(q, t) .
$$

The following proposition states the well-known properties of the function $P(q, t)(\mathrm{cf} .[\mathrm{F} 2, \mathrm{P}])$. 
Proposition 2.2.

(i) $P(q, t): \mathbb{R} \times \mathbb{R} \rightarrow \mathbb{R}$ is continuous.

(ii) $P(q, t)$ is strictly decreasing in each variable separately.

(iii) For fixed $q$ we have

$$
\lim _{t \rightarrow \infty} P(q, t)=-\infty \text { and } \lim _{t \rightarrow-\infty} P(q, t)=\infty .
$$

(iv) $P(q, t)$ is convex: if $q_{1}, q_{2}, t_{1}, t_{2} \in \mathbb{R}, a_{1}, a_{2} \geq 0, a_{1}+a_{2}=1$, then

$$
P\left(a_{1} q_{1}+a_{2} q_{2}, a_{1} t_{1}+a_{2} t_{2}\right) \leq a_{1} P\left(q_{1}, t_{1}\right)+a_{2} P\left(q_{2}, t_{2}\right) .
$$

For a given $q \in \mathbb{R}, P(q, t)$ is a continuous function of $t$. Its value ranges from $-\infty$ (when $t \rightarrow \infty$ ) to $\infty$ (when $t \rightarrow-\infty$ ). Therefore, by the intermediate value theorem there is a real number $\beta$ such that $P(q, \beta)=0$. The solution $\beta$ is unique, since $P(q, \cdot)$ is strictly decreasing. This defines $\beta$ implicitly as a function of $q$ : for each $q$ there is a unique $\beta=\beta(q)$ such that $P(q, \beta(q))=0$.

The following proposition gives the well-known properties of the function $\beta(q)$ (cf. [F2, $\mathrm{P}]$ ).

Proposition 2.3. Let $\beta=\beta(q)$ be defined by $P(q, \beta(q))=0$. Then

(i) $\beta$ is a continuous function of the real variable $q$.

(ii) $\beta$ is strictly decreasing: if $q_{1}<q_{2}$, then $\beta\left(q_{1}\right)>\beta\left(q_{2}\right)$.

(iii) $\lim _{q \rightarrow-\infty} \beta(q)=\infty$ and $\lim _{q \rightarrow \infty} \beta(q)=-\infty$.

(iv) $\beta$ is convex: if $q_{1}, q_{2}, a_{1}, a_{2} \in \mathbb{R}$ with $a_{1}, a_{2} \geq 0$ and $a_{1}+a_{2}=1$, then

$$
\beta\left(a_{1} q_{1}+a_{2} q_{2}\right) \leq a_{1} \beta\left(q_{1}\right)+a_{2} \beta\left(q_{2}\right) .
$$

The function $\beta(q)$ is sometimes denoted by $T(q)$ and called the temperature function. A more general discussion of this function can be found in [HJKPS], where our $\beta(q)$ corresponds to $-\tau(q)$ in their notation.

REMARK 2.4. If $q=0$, then $P(q, \beta(q))=0$ implies

$$
0=\lim _{k \rightarrow \infty} \frac{1}{k} \log \sum_{|\sigma|=k} s_{\sigma}^{\beta(0)}=\lim _{k \rightarrow \infty} \frac{1}{k} \log \left(\sum_{j=1}^{N} s_{j}^{\beta(0)}\right)^{k}=\log \sum_{j=1}^{N} s_{j}^{\beta(0)},
$$

and so

$$
\sum_{j=1}^{N} s_{j}^{\beta(0)}=1 .
$$

Hence, $\beta(0)$ gives the Hausdorff dimension $\operatorname{dim}_{H}(E)$ of the inhomogeneous self-similar set $E$. Note that

$$
P(1,0)=\lim _{k \rightarrow \infty} \frac{1}{k} \log \sum_{|\sigma|=k} \mu\left(E_{\sigma}\right)=\lim _{k \rightarrow \infty} \frac{1}{k} \log 1=0,
$$

and hence $\beta(1)=0$.

In the next section we state and prove the main result of the paper. 
3. Main result. The following theorem gives the bounds of the lower and the upper quantization dimensions of the attracting measure $\mu$ in terms of the quantization dimension of the self-similar measure $\nu$ and the temperature function $\beta(q)$.

THEOREM 3.1. Let $\mu$ be the attracting measure of the condensation system $\left(\left\{S_{j}\right\},\left\langle p_{j}\right\rangle, \nu\right)$, where $\left\{S_{1}, \ldots, S_{N}\right\}$ satisfies the strong separation condition, and $\nu$ is a self-similar measure. Let $\beta=\beta(q)$ be the temperature function of the thermodynamic formalism for the attracting measure $\mu$. For each $r \in(0, \infty)$ choose $q_{r}$ such that $\beta\left(q_{r}\right)=r q_{r}$. Then the lower and the upper quantization dimensions $\underline{D}_{r}(\mu)$ and $\bar{D}_{r}(\mu)$ of order $r$ of the attracting measure $\mu$ satisfy

$$
D_{r}(\nu) \leq \underline{D}_{r}(\mu) \leq \bar{D}_{r}(\mu) \leq \frac{\beta\left(q_{r}\right)}{1-q_{r}},
$$

where $D_{r}(\nu)$ is the quantization dimension of order $r$ of $\nu$.

To prove the theorem we need some lemmas and propositions. The following lemma plays a vital role.

LEMma 3.2. Let $0<r<\infty$ be fixed. Then there exists exactly one $\kappa_{r} \in(0, \infty)$ such that

$$
\lim _{k \rightarrow \infty} \frac{1}{k} \log \sum_{|\sigma|=k}\left(\mu\left(E_{\sigma}\right) s_{\sigma}^{r}\right)^{\kappa_{r} /\left(r+\kappa_{r}\right)}=0 .
$$

Proof. By (5) we have

$$
P(t, r t)=\lim _{k \rightarrow \infty} \frac{1}{k} \log \sum_{|\sigma|=k}\left(\mu\left(E_{\sigma}\right) s_{\sigma}^{r}\right)^{t} .
$$

If $t=0$, then

$$
P(0,0)=\lim _{k \rightarrow \infty} \frac{1}{k} \log \sum_{|\sigma|=k} 1=\lim _{k \rightarrow \infty} \frac{1}{k} \log N^{k}=\log N>0 ;
$$

and if $t=1$,

$$
\begin{aligned}
P(1, r 1) & =\lim _{k \rightarrow \infty} \frac{1}{k} \log \sum_{|\sigma|=k} \mu\left(E_{\sigma}\right) s_{\sigma}^{r} \leq \lim _{k \rightarrow \infty} \frac{1}{k} \log \sum_{|\sigma|=k} \mu\left(E_{\sigma}\right) s_{\max }^{k r} \\
& =\lim _{k \rightarrow \infty} \frac{1}{k} \log \sum_{|\sigma|=k} \mu\left(E_{\sigma}\right)+r \log s_{\max } \\
& =r \log s_{\max }<0 .
\end{aligned}
$$

Since $P(t, r t)$ is continuous and strictly decreasing, the unique $t \in \mathbb{R}$ for which $P(t, r t)=0$ must lie between 0 and 1 . Then $\kappa_{r}=r t /(1-t)$ satisfies the conclusion of the lemma. 
Let us now give the following lemma.

Lemma 3.3. For any $\tau, \sigma \in \Xi_{*}$, we have

$$
\mu\left(E_{\tau \sigma}\right) \geq p_{0} \mu\left(E_{\tau}\right) \nu\left(E_{\sigma}\right) .
$$

Proof. As shown in the proof of Lemma 2.1. we have $\mu\left(E_{\sigma}\right) \geq p_{0} \nu\left(E_{\sigma}\right)$ for all $\sigma \in \Xi_{*}$. Thus,

$$
\mu\left(E_{\tau \sigma}\right)=g^{(1)}(\tau) \nu\left(E_{\sigma}\right)+g^{(2)}(\tau) \mu\left(E_{\sigma}\right) \geq g^{(1)}(\tau) \nu\left(E_{\sigma}\right)+g^{(2)}(\tau) p_{0} \nu\left(E_{\sigma}\right),
$$

which implies

$$
\mu\left(E_{\tau \sigma}\right) \geq p_{0}\left(g^{(1)}(\tau)+g^{(2)}(\tau)\right) \nu\left(E_{\sigma}\right)=p_{0} \mu\left(E_{\tau}\right) \nu\left(E_{\sigma}\right) .
$$

From the above lemma the following corollary can easily be deduced.

Corollary 3.4 (cf. [Zh, Lemma 1]). Let $0<r<\infty$ be fixed. Then there exists a constant $0<C<1$ such that for all $\sigma \in \Xi_{*}$, we have

$$
\mu\left(E_{\sigma}\right) s_{\sigma}^{r} \geq C \mu\left(E_{\sigma^{-}}\right) s_{\sigma^{-}}^{r} .
$$

Proof. Let $L=\min \left\{t_{1} s_{1}^{r}, \ldots, t_{N} s_{N}^{r}\right\}$. Write $C=p_{0} L$; then $0<C<1$. Using the above lemma, we have

$$
\mu\left(E_{\sigma}\right) s_{\sigma}^{r} \geq p_{0} \mu\left(E_{\sigma^{-}}\right) \nu\left(E_{\sigma_{|\sigma|}}\right) s_{\sigma^{-}}^{r} s_{\sigma_{|\sigma|}}^{r} \geq C \mu\left(E_{\sigma^{-}}\right) s_{\sigma^{-}}^{r} .
$$

We call $\Gamma \subset \Xi_{*}$ a finite maximal antichain if $\Gamma$ is a finite set of words in $\Xi_{*}$ such that every sequence in $\Xi$ is an extension of some word in $\Gamma$, but no word of $\Gamma$ is an extension of another word in $\Gamma$. Of course, this requires that the index set $\{1, \ldots, N\}$ is finite. We will make this assumption in the remainder of this paper. By $|\Gamma|$ we denote the cardinality of $\Gamma$. Note that from the definition of $\Gamma$ it follows that no finite maximal antichain contains the empty word $\emptyset$ as all words are extensions of $\emptyset$.

Let us now state the following two lemmas.

Lemma 3.5 (cf. [Zh, Lemma 4]). Let $\Gamma \subset \Xi_{*}$ be a finite maximal antichain, $n \in \mathbb{N}$ with $n \geq|\Gamma|$, and $0<r<\infty$. Then for any sequence $\left\{n_{\sigma}: \sigma \in \Gamma\right\}$ of natural numbers satisfying $n_{\sigma} \geq 1, \sum_{\sigma \in \Gamma} n_{\sigma} \leq n$, we have

$$
V_{2 n, r}(\mu) \leq \sum_{\sigma \in \Gamma}\left(g^{(1)}(\sigma) s_{\sigma}^{r} V_{n_{\sigma}, r}(\nu)+g^{(2)}(\sigma) s_{\sigma}^{r} V_{n_{\sigma}, r}(\mu)\right) .
$$

Lemma 3.6 (cf. [Zh, Lemma 5]). There exists a constant $D>1$ such that $V_{n, r}(\nu) \leq D V_{n, r}(\mu)$ for all $n \geq 1$.

REMARK 3.7. Let $0<r<\infty$ be fixed and $\kappa_{r}$ be as in Lemma 3.2. We assume that there exists a constant $C_{r}>1$ such that for any finite maximal antichain $\Gamma$,

$$
\sum_{\sigma \in \Gamma}\left(\mu\left(E_{\sigma}\right) s_{\sigma}^{r}\right)^{\kappa_{r} /\left(r+\kappa_{r}\right)} \leq C_{r} .
$$

This assumption is needed to prove the following proposition. 
Proposition 3.8. Let $0<r<\infty$ be fixed and $\kappa_{r}$ be as in Lemma 3.2 . Then under the assumption of Remark 3.7, we have

$$
\limsup _{n \rightarrow \infty} n V_{n, r}^{\kappa_{r} / r}(\mu)<\infty .
$$

Proof. Let $0<C<1$ be the constant as defined in Corollary 3.4 , and $C_{r}$ be as in Remark 3.7. Fix $m \in \mathbb{N}$. Choose any $n \in \mathbb{N}$ so that $m / n<C C_{r}^{-1}$, and set $\epsilon=C_{r} C^{-1} m / n$. Then $0<\epsilon<1$. Let

$$
\Gamma=\Gamma(\epsilon)=\left\{\sigma \in \Xi_{*}:\left(\mu\left(E_{\sigma}\right) s_{\sigma}^{r}\right)^{\kappa_{r} /\left(r+\kappa_{r}\right)}<\epsilon \leq\left(\mu\left(E_{\sigma^{-}}\right) s_{\sigma^{-}}^{r}\right)^{\kappa_{r} /\left(r+\kappa_{r}\right)}\right\} .
$$

Then by Remark 3.7 and Corollary 3.4 , we have

$$
C_{r} \geq \sum_{\sigma \in \Gamma}\left(\mu\left(E_{\sigma}\right) s_{\sigma}^{r}\right)^{\kappa_{r} /\left(r+\kappa_{r}\right)} \geq C^{\kappa_{r} /\left(r+\kappa_{r}\right)} \sum_{\sigma \in \Gamma}\left(\mu\left(E_{\sigma^{-}}\right) s_{\sigma^{-}}^{r}\right)^{\kappa_{r} /\left(r+\kappa_{r}\right)}>C \epsilon|\Gamma|,
$$

which implies $|\Gamma|<C_{r}(C \epsilon)^{-1}=n / m<\infty$, i.e., $\Gamma$ is a finite maximal antichain and $n>|\Gamma| m$. Hence by Lemmas 3.5 and 3.6 , we have

$$
\begin{aligned}
V_{2 n, r}(\mu) & \leq \sum_{\sigma \in \Gamma}\left(g^{(1)}(\sigma) s_{\sigma}^{r} V_{m, r}(\nu)+g^{(2)}(\sigma) s_{\sigma}^{r} V_{m, r}(\mu)\right) \\
& \leq D \sum_{\sigma \in \Gamma} \mu\left(E_{\sigma}\right) s_{\sigma}^{r} V_{m, r}(\mu) \\
& =D \sum_{\sigma \in \Gamma}\left(\mu\left(E_{\sigma}\right) s_{\sigma}^{r}\right)^{\kappa_{r} /\left(r+\kappa_{r}\right)}\left(\mu\left(E_{\sigma}\right) s_{\sigma}^{r}\right)^{r /\left(r+\kappa_{r}\right)} V_{m, r}(\mu),
\end{aligned}
$$

which implies

$$
\begin{aligned}
V_{2 n, r}(\mu) & \leq D \sum_{\sigma \in \Gamma}\left(\mu\left(E_{\sigma}\right) s_{\sigma}^{r}\right)^{\kappa_{r} /\left(r+\kappa_{r}\right)} \epsilon^{r / \kappa_{r}} V_{m, r}(\mu) \leq D C_{r} \epsilon^{r / \kappa_{r}} V_{m, r}(\mu) \\
& =D C_{r}\left(C_{r} C^{-1}\right)^{r / \kappa_{r}}\left(\frac{m}{n}\right)^{r / \kappa_{r}} V_{m, r}(\mu),
\end{aligned}
$$

yielding

$$
2 n V_{2 n, r}^{\kappa_{r} / r}(\mu) \leq 2\left(D C_{r}\right)^{\kappa_{r} / r} C_{r} C^{-1} m V_{m, r}^{\kappa_{r} / r}(\mu) .
$$

Since for fixed $m$, this inequality holds for all but a finite number of $n$, we have

$$
\limsup _{n \rightarrow \infty} 2 n V_{2 n, r}^{\kappa_{r} / r}(\mu) \leq 2\left(D C_{r}\right)^{\kappa_{r} / r} C_{r} C^{-1} m V_{m, r}^{\kappa_{r} / r}(\mu)<\infty .
$$

Let us now prove the following proposition.

Proposition 3.9. Let the similarity mappings $\left\{S_{j}: 1 \leq j \leq N\right\}$ satisfy the strong separation condition and let $0<r<\infty$. Moreover, let $D_{r}(\nu)$ be the quantization dimension of order $r$ of the self-similar measure $\nu$. Then

$$
\liminf _{n \rightarrow \infty} n e_{n, r}^{D_{r}(\nu)}(\mu)>0 .
$$


Proof. Since $D_{r}(\nu)$ is the quantization dimension of order $r$ of the selfsimilar measure $\nu$, by (2) it follows that $\liminf _{n \rightarrow \infty} n e_{n, r}^{D_{r}(\nu)}(\nu)>0$. By Lemma 3.6, for any $n \geq 1$, we have

$$
e_{n, r}(\nu)=V_{n, r}^{1 / r}(\nu) \leq D^{1 / r} V_{n, r}^{1 / r}(\mu)=D^{1 / r} e_{n, r}(\mu) .
$$

Hence,

$$
\liminf _{n \rightarrow \infty} n e_{n, r}^{D_{r}(\nu)}(\mu) \geq D^{-D_{r}(\nu) / r} \liminf _{n \rightarrow \infty} n e_{n, r}^{D_{r}(\nu)}(\nu)>0 .
$$

Proof of Theorem 3.1. By Proposition 11.3 of [GL1], we know that:

(a) If $0 \leq t<\underline{D}_{r}<s$ then

$$
\lim _{n \rightarrow \infty} n e_{n, r}^{t}=\infty \quad \text { and } \quad \liminf _{n \rightarrow \infty} n e_{n, r}^{s}=0 .
$$

(b) If $0 \leq t<\bar{D}_{r}<s$ then

$$
\limsup _{n \rightarrow \infty} n e_{n, r}^{t}=\infty \quad \text { and } \quad \lim _{n \rightarrow \infty} n e_{n, r}^{s}=0 .
$$

By (a) and Proposition 3.9, we have $D_{r}(\nu) \leq \underline{D}_{r}(\mu)$. By (b) and Proposition 3.8, we have $\bar{D}_{r}(\mu) \leq \kappa_{r}$. Hence, $D_{r}(\nu) \leq \underline{D}_{r}(\mu) \leq \bar{D}_{r}(\mu) \leq \kappa_{r}$. Note that if $q_{r}=\kappa_{r} /\left(r+\kappa_{r}\right)$ then by Lemma 3.2, $\beta\left(q_{r}\right)=r q_{r}$. Thus it follows that $\kappa_{r}=\beta\left(q_{r}\right) /\left(1-q_{r}\right)$, proving the theorem.

REMARK 3.10. If $p_{0} t_{j}+p_{j}=t_{j}$ for $1 \leq j \leq N$, then the inhomogeneous self-similar measure $\mu$ reduces to the self-similar measure $\nu$, i.e., $\mu=\nu$ (for the proof see [Zh, Proposition 1]). Then by Lemma 3.2, we have

$$
\begin{aligned}
0 & =\lim _{k \rightarrow \infty} \frac{1}{k} \log \sum_{|\sigma|=k}\left(t_{\sigma} s_{\sigma}^{r}\right)^{\kappa_{r} /\left(r+\kappa_{r}\right)}=\lim _{k \rightarrow \infty} \frac{1}{k} \log \left(\sum_{j=1}^{N}\left(t_{j} s_{j}^{r}\right)^{\kappa_{r} /\left(r+\kappa_{r}\right)}\right)^{k} \\
& =\log \left(\sum_{j=1}^{N}\left(t_{j} s_{j}^{r}\right)^{\kappa_{r} /\left(r+\kappa_{r}\right)}\right),
\end{aligned}
$$

and so

$$
\sum_{j=1}^{N}\left(t_{j} s_{j}^{r}\right)^{\kappa_{r} /\left(r+\kappa_{r}\right)}=1 .
$$

Graf and Luschgy showed that the above $\kappa_{r}$ is the quantization dimension of order $r$ of the self-similar measure $\nu$, i.e., $D_{r}(\nu)=\kappa_{r}$ (cf. [GL1, GL2]). Thus, if $p_{0} t_{j}+p_{j}=t_{j}$ for $1 \leq j \leq N$, then our result reduces to $D_{r}(\nu)=$ $\underline{D}_{r}(\mu)=\bar{D}_{r}(\mu)=\kappa_{r}$, i.e., $D_{r}(\nu)=D_{r}(\mu)=\kappa_{r}$.

Acknowledgements. The author would like to express his sincere thanks and gratitude to the referee for the valuable comments and suggestions. 


\section{References}

[B] M. R. Barnsley, Fractals Everywhere, Academic Press, New York, 1988.

[F1] K. J. Falconer, Techniques in Fractal Geometry, Wiley, Chichester, 1997.

[F2] K. J. Falconer, The multifractal spectrum of statistically self-similar measures, J. Theoret. Probab. 7 (1994), 681-701.

[GL1] S. Graf and H. Luschgy, Foundations of Quantization for Probability Distributions, Lecture Notes in Math. 1730, Springer, Berlin, 2000.

[GL2] S. Graf and H. Luschgy, The quantization dimension of self-similar probabilities, Math. Nachr. 241 (2002), 103-109.

[GN] R. Gray and D. Neuhoff, Quantization, IEEE Trans. Inform. Theory 44 (1998), 2325-2383.

[HJKPS] T. Halsey, M. Jensen, L. Kadanoff, I. Procaccia and B. Shraiman, Fractal measures and their singularities: the characterization of strange sets, Phys. Rev. A 33 (1986), 1141-1151; erratum, ibid. 34 (1986), 1601.

[H] J. Hutchinson, Fractals and self-similarity, Indiana Univ. Math. J. 30 (1981), 713-747.

[L] A. Lasota, A variational principle for fractal dimensions, Nonlinear Anal. 64 (2006), 618-628.

[OS1] L. Olsen and N. Snigireva, $L^{q}$ spectra and Rényi dimensions of in-homogeneous self-similar measures, Nonlinearity 20 (2007), 151-175.

[OS2] L. Olsen and N. Snigireva, In-homogenous self-similar measures and their Fourier transforms, Math. Proc. Cambridge Philos. Soc. 144 (2008), 465-493.

[OS3] L. Olsen and N. Snigireva, Multifractal spectra of in-homogeneous self-similar measures, Indiana Univ. Math. J. 57 (2008), 1789-1843.

[P] N. Patzschke, Self-conformal multifractal measures, Adv. Appl. Math. 19 (1997), 486-513.

[S] A. Schief, Separation properties for self-similar sets, Proc. Amer. Math. Soc. 122 (1994), 111-115.

[Za] P. L. Zador, Asymptotic quantization error of continuous signals and the quantization dimension, IEEE Trans. Inform. Theory 28 (1982), 139-149.

[Zh] S. Zhu, Quantization dimension for condensation systems, Math. Z. 259 (2008), $33-43$.

Mrinal Kanti Roychowdhury

Department of Mathematics

The University of Texas - Pan American

1201 West University Drive

Edinburg, TX 78539-2999, U.S.A.

E-mail: roychowdhurymk@utpa.edu

Received July 20, 2012;

received in final form December 22, 2012

(7893) 
